\title{
Two-Dimensional Nucleation with Edge and Corner Diffusion
}

\author{
Yukio Saitd* \\ Department of Physics, Keio University, 3-14-1 Hiyoshi, Kohoku-ku, Yokohama 223-8522, Japan
}

(Dated: October 28, 2018)

\begin{abstract}
The effect of edge and corner diffusions on the morphology and on the density of islands nucleated irreversibly on a flat substrate surface is studied. Without edge and corner diffusion, islands are fractal. As an edge diffusion constant $D_{e}$ increases, islands tend to take a cross shape with four needles in the $\langle 10\rangle$ direction. Additional corner diffusion with a diffusion constant $D_{c}$ yields square islands. When $D_{e}$ is small relative to the surface diffusion constant $D_{s}$, the square corner shows the Berg instability to produce hopper growth in the $<11\rangle$ direction. The corner diffusion influences the island number density $n$. At a deposition flux $F$ with a small $D_{c}$, mainly monomers are mobile and $n \propto\left(F / D_{s}\right)^{1 / 3}$. At large $D_{c}$, dimers and trimers are also mobile and $n \propto F^{3 / 7} D_{s}^{-5 / 21} D_{c}^{-4 / 21}$. The $F$ dependence is in good agreement to the rate equation analysis, but the dependence on $D_{c}$ cannot be explained by the theory.
\end{abstract}

PACS numbers: 68.55.-a, 68.43.Jk, 05.70.Ln, 81.10.Aj

\section{INTRODUCTION}

Surface morphology during epitaxial growth depends on processes taking place on the substrate 1.2.3.4.5.6.7.8 At very low temperatures as in the case of molecular beam epitaxy (MBE), deposited atoms are adsorbed and migrate on the substrate surface until they form nuclei or are incorporated into islands, but they never evaporate back to the ambient vapor. When an adsorbed atom (adatom, for short) sticks and freezes irreversibly to an island, the resulting aggregate takes an irregular dendritic form, 1.2.4.5.6 called diffusion-limited aggregate (DLA) $\stackrel{9}{9}$ It has a characteristic feature of self-similarity, and is regarded as a typical example of a fractal. In actual experiments, one does not always observe fractal but also compact islands $\stackrel{2.3}{ }$ Compact islands are possible if they can dissociate $\frac{10}{}$ or if atoms at the edge of them can diffuse along the periphery 2.8 .11 .12 .13 .14 .15 .16 Since at low temperatures it is difficult for an edge atom to detach, the edge diffusion mainly governs the island morphology 8 During the edge diffusion, however, the outer corner provides an additional barrier to be surpassed. There, an edge atom has to pass an intermediate state where the number of neighboring bonds is less than that on the straight step edge. The edge and the corner diffusion is known to influence island morphology $2,6,7,8,11,12,13,14,15,16$ Without edge and corner diffusion, islands are similar to DLA. With the edge diffusion they take cross shapes, with the corner diffusion in addition they become square. At the coalescence, these peripheral diffusion governs the shape relaxation 17 Furthermore, the corner diffusion barrier is found to induce mound formation for multilayer deposition. ${ }^{18,19}$

Diffusion processes are also known to influence the number density $n$ of islands nucleated on a singular surface. $20,21,22,23,24,25,26$ When the deposition rate $F$ is large, the adatom density is high and the nucleation occurs frequently. Thus the island density $n$ is expected to be high. When an isolated adatom migrates on the surface with a large surface diffusion constant $D_{s}$, the de- posited atom moves around a long distance, until it finds nucleation partners or preexisting islands and sticks to them. Thus, as the diffusion rate $D_{s}$ increases, the rate of forming new nuclei decreases, and the island density $n$ on a singular surface diminishes.

More quantitatively, the island density $n$ is defined as the total number density of immobile and undissociable clusters with sizes larger than the critical cluster size $i^{*}$. In the classical mean-field nucleation theory, the number density of adatom clusters of size $i$ is analyzed by means of rate equations ${ }^{20,21,24,25,27}$ When clusters smaller than $i^{*}$ can dissociate but only the mononer adatom is mobile with the diffusion constant $D_{s}, n$ is found to follow the scaling law ${ }^{20.21}$

$$
n \propto\left(\frac{F}{D_{s}}\right)^{i^{*} /\left(i^{*}+2\right)} .
$$

If the clusters with sizes $i$ smaller than the critical size $i^{*}$ cannot dissociate but are mobile as a whole with their respective diffusion constant $D_{i}$, the scaling form is approximately given $\operatorname{as}^{24.25 .27}$

$$
n \propto\left(\frac{F^{i^{*}}}{D_{s} D_{2} D_{3} \cdots D_{i^{*}}}\right)^{1 /\left(2 i^{*}+1\right)} .
$$

By assuming that islands are point sinks to diffusing adatoms and the critical nuclear size is $i^{*}=1$, the island density scaling $n \propto\left(F / D_{s}\right)^{1 / 3}$ is confirmed by the kinetic Monte Carlo (KMC) simulation. ${ }^{28}$ Actually, islands increase their size during growth, and the scaling relation between the density and the diffusion constant might depend on the island morphology. There are already many studies on the island density by means of KMC simulation when clustes can dissociate. $8.11,29,30$ There are also KMC simulations with mobile clusters. 27,29,30.31 We study here the effect of edge and corner diffusion processes on the island density and its relation to the island morphology, systematically.

In $\S 2$, we explain our simulation scheme. In $\S 3$, the effect of peripheral diffusion on the island morphology 
is studied. On varying the ratio between the surface, edge and corner diffusion, morphology varies as fractal, needle, and square shapes, as previously observed 16 We point out the different roles of the edge and corner diffusions on the island symmetry. The island density is discussed in $\S 4$. As long as the corner diffusion is small, the density scaling is found to be almost independent of the island morphology with an exponent $1 / 3$, in consistent to the more systematic study by Brune et al $\stackrel{8}{*}$ With a large corner diffusion constant, on the other hand, the scaling form of the island density changes drastically, because small clusters of sizes less than 3 can migrate on the surface, i.e. $i^{*}=3$. The density is well fitted to the scaling $n \sim F^{3 / 7}$. The increase of the critical island size $i^{*}$ is also reflected in the island size distribution. ${ }^{27.31 .32}$ The result is summarized in a scaling plot of the island density, and discussed in the last $\S 5$. Even though the scaling of the island density to the deposition flux agrees to the rate equation analysis, the dependence on the diffusion constants fails to follow the expectation (2).

\section{MONTE CARLO SIMULATION}

Our main aim is to explore the systematics and the universal features of the morphology change of islands on a singular surface as the diffusion mechanism changes, rather than the precise reproduction of some specific experimental observations. For this purpose, we adopt the simplest model such that atoms are depositing on a (100) surface of a simple cubic lattice with a solid-on-solid (SOS) restriction. The SOS restriction is valid at low temperatures, common to MBE.

Atoms are deposited randomly onto the substrate with a deposition rate of $F$ monolayers per unit time. Here we choose the unit of time such that $F=1$. The temperature is assumed to be so low that all deposited atoms stick to the surface and never evaporate back into the ambient gas. An isolated adatom hops to one of its four nearest neighboring sites at a rate $k_{s}$; the waiting time of an adatom before the jump is $\left(4 k_{s}\right)^{-1}$, and the surface diffusion constant is given by $D_{s}=k_{s} a^{2}$, where $a$ is the lattice constant. As for the interlayer diffusion, which takes place in case a deposited atom lands on another adatom, no additional energy barrier is assumed for it to diffuse down. On the contrary, atoms at the edge are not allowed to hop up a layer. Therefore, Ehrlich-Schwoebel mound formation is supressed, and the layer-by-layer growth is expected.

When an atom touches to other adatoms or islands, it makes a nearest neighbor bond to form a cluster. At very low temperatures, this cluster will never dissociate again. We assume that adatoms attach clusters irreversibly, and no cluster dissociation takes place. This means that dimers are stable, and the critical island size is expected to be $i^{*}=1$. Nevertherless, if an edge atom is singly-bonded in the layer, i.e. if it makes only one bond with another adatom, it is possible to diffuse along the

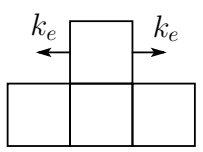

(a) an ee atom

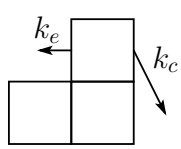

(b) an ec atom

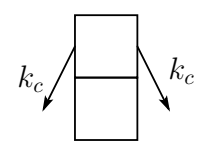

(c) a $c c$ atom
FIG. 1: Diffusion processes of an adatom connected to an island with a single in-layer bond. $k_{e}$ is the rate of edge diffusion and $k_{c}$ is that of corner diffusion.

island periphery at moderate temperatures. There are two possible diffusion jumps for a singly-bonded atom; an edge diffusion along the straight step edge, and a corner diffusion or corner crossing round the outer corner. The rate of edge diffusion is $k_{e}$ and that of corner is $k_{c}$, as is depicted in Fig 1] Then, a singly-bonded atom can be classified by its possible motions in two directions. It is classified as (a) an ee atom on a straight step if both movements are along the step edge, (b) an $e c$ atom at the ridge of a corner if one is the edge diffusion and the other is the corner, and (c) a $c c$ atom on a tip if both movements are across the corner, as shown in Fig 1 The edge diffusion constant along the straight step edge is $D_{e}=k_{e} a^{2}$, and we may define the corner diffusion constant as $D_{c}=k_{c} a^{2}$. Precise values of diffusion constants depend on the energy barriers and the temperature. For edge and corner diffusion there may exist extra energy barriers in addition to that for the surface diffusion, and diffusion constants are probably ordered as $D_{s} \geq D_{e} \geq D_{c} \geq 0$. If an edge atom is incoorporated into the kink site with more than two nearest neighbors, it is assumed to cease migration, for simplicity.

Simulation starts from a clean substrate surface of a size $L \times L$ without adatoms. Hereafter we take the lattice constant $a=1$. Therefore, the jump rates $k$ 's and diffusion constants $D$ 's are the same. During the simulation, at some stage, there are $N_{0}$ isolated adatoms, $N_{e e}$ edge atoms attached to a straight step edge, $N_{e c}$ atoms at the corner, and $N_{c c}$ atoms at the tip position (see Fig (1) on the surface. These adatoms are sorted out in corresponding lists. The transition probability from this configuration to the next one is given as follows. The rate of the deposition is $P_{d}=F L^{2}$, of the surface diffusion $P_{s}=4 N_{0} D_{s}$, of the edge diffusion $P_{e}=\left(2 N_{e e}+N_{e c}\right) D_{e}$ and of the corner diffusion $P_{c}=\left(N_{e c}+2 N_{c c}\right) D_{c}$. The total probability of a state change in a unit of time is $P_{t}=P_{d}+P_{s}+P_{e}+P_{c}$. Thus, within a time interval $d t=1 / P_{t}$, one of the events takes place; the deposition with a probability $P_{d} d t$, the surface diffusion with $P_{s} d t$, the edge diffusion with $P_{e} d t$ and the corner diffusion with $P_{c} d t$. For each diffusion process, the atom to be moved is picked up randomly from the list. After each state change, adatom lists have to be adjusted, for example, by eliminating those adatoms with more than two neighboring bonds from the lists, or add an adatom that happens to have a single bond to the corresponding list. The time is increased by $d t$. Since the time unit is chosen such that 
the deposition flux $F=1$, the monolayer is covered at a time $t=1$, in principle. Because of the stochastic feature of the simulation algorithm, in practice, the actual time for the completion of a monolayer fluctuates around $t=1$.

\section{ISLAND MORPHOLOGY}

Simulations are performed for systems of sizes $200 \times$ 200 , and $1000 \times 1000$ under various combinations of diffusion constants, and some typical island morphology is shown in Fig 2 at a coverage of $\theta=0.1$ monolayer(ML).

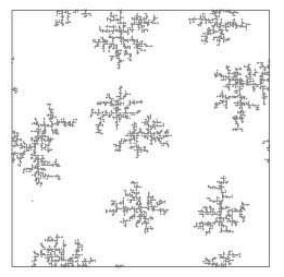

(a) $(0,0)$

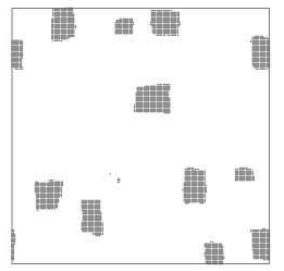

(d) $\left(10^{-4}, 10^{-4}\right)$

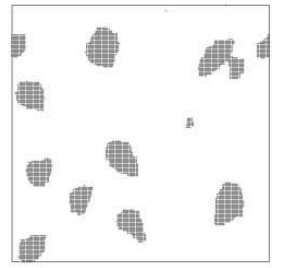

(g) $\left(1,10^{-5}\right)$

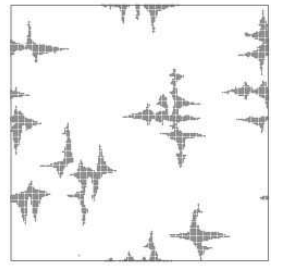

(j) $\left(10^{-4}, 0\right)$

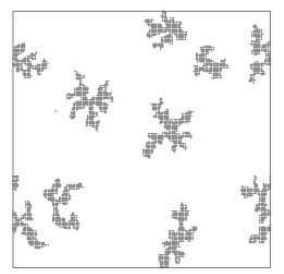

(b) $\left(10^{-6}, 10^{-6}\right)$

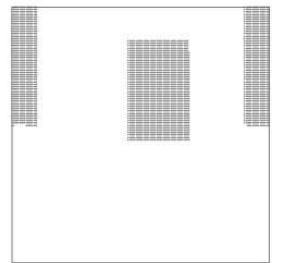

(e) $(1,1)$

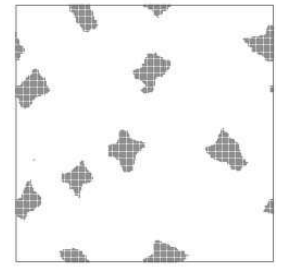

(h) $\left(1,10^{-6}\right)$

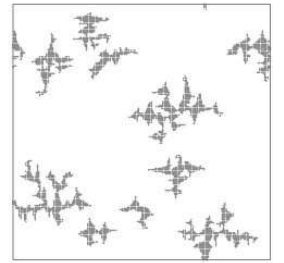

(k) $\left(10^{-5}, 0\right)$
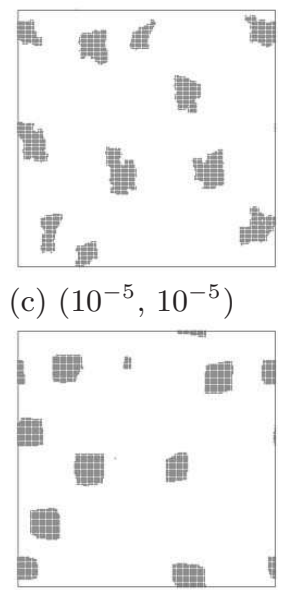

(f) $\left(1,10^{-4}\right)$

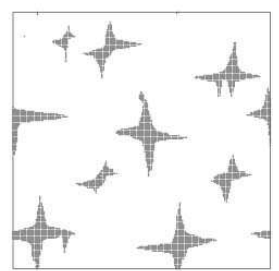

(i) $(1,0)$

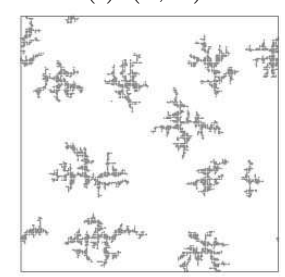

(1) $\left(10^{-6}, 0\right)$
FIG. 2: Island morphology at various combinations of the surface, edge and corner diffusion constants, $D_{s}, D_{e}$ and $D_{c}$. $D_{s}$ is fixed at $D_{s} / F=10^{9}$. Parameters in parenthes represents $\left(D_{e} / D_{s}, D_{c} / D_{s}\right)$. The system size is $200 \times 200$, and the coverage is $\theta=0.1 \mathrm{ML}$.

From Fig 2(a) to (e) the edge and corner diffusion constants, $D_{e}$ and $D_{c}$, are equally increased from 0 to $D_{s}$, by keeping the surface diffusion constant $D_{s}$ fixed at $D_{s} / F=10^{9}$. Without peripheral diffusion, the island takes an irregular dendritic form or a fractal DLAlike shape, as shown in Fig 2(a). On increasing the edge and corner diffusion to $D_{e}=D_{c}=10^{-6} D_{s}$, dendrite branchs thicken and they extend mainly in the $<11>$ direction, as shown in Fig 2(b). Further increase of $D_{e}=D_{c}=10^{-5} D_{s}$ let the islands compact in a hopper shape with a corner instability due to Berg effect, $\stackrel{33}{3}$ as in Fig 2(c). Since the corner is extending in the region with high monomer concentration, the corner captures many adatoms and the edge diffusion transfers them to the island center. But the edge diffusion is still weak and insufficient, and the growth of the central part is too slow to catch up the fast corner growth. Thus, the island grows mainly in the $\langle 11\rangle$ direction, as in Fig 2(b) and (c). With sufficient edge and corner diffusion as $D_{e}=D_{c}=10^{-4} D_{s}$, islands take square shape surrounded by rather straight $\{10\}$ steps, as in Fig 2 (d). So far island density seems independent on the island morphology. When all the diffusion constants are equal, as in Fig[2(e), islands are square and very large, but their density decreases drastically. On this density change we shall discuss later in $\S 4$. The sequence of simulations from Fig 2(b) to (d) almost corresponds to the parameter range studied by Bales and Chrzan 11 by changing the temperature. The morphology variation is of course quite analogous to what they have obtained.

We now study the effect of corner diffusion more precisely, in a sequence of pictures shown from Fig 2 (e) to (i). There, the edge diffusion constant is kept as large as $D_{e}=D_{s}$, and only the corner diffusion constant $D_{c}$ is decreased from $D_{s}$ to 0 . In all these cases, islands are not irregular. With a large corner diffusion constant, $D_{c} \geq 10^{-4} D_{s}$, island are square with $\{10\}$ steps, as shown in Fig 2(e) and (f). At $D_{c}=10^{-5} D_{s}$ in Fig $2(\mathrm{~g})$, they look round and rather isotropic, and for a still smaller $D_{c}=10^{-6} D_{s}$ in Fig 2(h) some islands are in diamond shape and some others are in a cross shape with thick arms. In the extreme case of Fig 2 i) without the corner diffusion, $D_{c}=0$, islands consist four sharp needles growing in the $\langle 10\rangle$ direction. The same fourneedle shape is observed previously by Zhong et al ${ }^{16}$ in a submonolayer growth and by Caspersen et $\mathrm{al}^{34}$ in a multilayer study.

In a series of simulations Fig[2(i)-(l) the corner diffusion is completely suppressed $\left(D_{c}=0\right)$, and the effect of the edge diffusion $D_{e}$ is exemplified. The series, in fact, continues back to Fig 2(a). With $D_{e}=D_{s}$ in Fig 2(i) needles in cross-shape islands are rather stable and sidebranches are rare. On decreasing the edge diffusion to $D_{e}=10^{-4} D_{s}$ in Fig $2(\mathrm{j})$, needles have many sidebranches growing in the $\langle 10\rangle$ direction. Further decrement to $D_{e}=10^{-5} D_{s}$ leads to tip splitting in Fig.2(k). With still smaller $D_{e}=10^{-6} D_{s}$ clusters look like DLA aggregates. Comparison with Fig 2(b) and (l) reveals that the corner diffusion produces $<11\rangle$ dendrite in Fig 2(b), whereas without corner diffusion aggregates have a preference in the $\langle 10\rangle$ direction in Fig[2(1). Also without corner diffusion, the dendrite tip is pointed, whereas with a corner diffusion, the tip becomes fat. At $D_{e}=D_{c}=0$, the DLA looks isotropic. Of 
course, it is known to be anisotropic asymptotically by reflecting the underlying square symmetry of the lattice, but the shape anisotropy appears only when the DLA grows very large. In the present simulation, islands coagulate to acomplish a layer growth before the asymptotic anisotropy appears.

From these variety of island morphology the edge and corner diffusions are found to play different roles on the island symmetry. The edge diffusion smears out shot noise introduced by the irreversible attachment, and permits the formation of regular shape. The corner diffusion varies the orientational preference. Without the corner diffusion, those atoms on the tip positions or on the narrow step edges cannot cross to the other sides of an island, and thus the supersaturation on the narrow $\{10\}$ side increases. This leads to the enhancement in the growth velocity normal to the narrow $\{10\}$ side, and due to the strongly anisotropic kinetic effect a needle tip is eventually formed into the $\langle 10\rangle$ direction. A corner diffusion allows the transfer of adatoms from one step edge to the other, and islands tend to be square. If the edge diffusion is weak, however, the adatom supply at the center of the step edge is meager, and the corner instability takes place. This leads to the dendrite growth in the diagonal $<11>$ direction, as in Fig[2] and (c).

\section{ISLAND DENSITY}

In Fig 2 at a fixed surface diffusion constant $D_{s}$, we observe that the island density is almost independent of the edge and corer diffusion constants, $D_{e}$ and $D_{c}$. We said "almost", because in Fig 2(e) the island density is clearly different from the others. We now study the effect of peripheral diffusion on the island density.

The rate equation analysis predicts that the island density $n$ depends on the surface diffusion constant as in eqs. (11) and (2) $20,21,24,25$ Experimentally, temperature dependence of the island density is used to estimate values of various energy barriers by assuming the critical island size $i^{*}{ }^{22}$ Simulations where the temperature is varied also has to assume $i^{*}$ to interpret their results $\frac{22}{2}$ To study the relation of island morphology and its density by fixing $i^{*}=1$, we control directly on the rate of edge and corner diffusion, rather than tune many parameters as bond energies, energy barriers and the temperature.

The island density $n$ at the coverage $\theta=0.1 \mathrm{ML}$ is plotted in Fig 3 as a function of the surface diffusion constant $D_{s} / F$ for various combinations of the edge and corner diffusion constants. The system sizes plotted are $200^{2}$ (open circles) and $1000^{2}$ (filled circles).

For the case without edge and corner diffusion, $D_{e}=$ $D_{c}=0$, islands are fractal as shown in Fig 2(a), at least at low densities with a large $D_{s}$. The island density is shown in Fig 3(b). Data fits well with the scaling relation $n=0.23\left(D_{s} / F\right)^{-1 / 3}$. The result is consistent to that obtained by Venables et al ${ }^{22} n=0.25\left(D_{s} / F\right)^{-1 / 3}$ from the rate equation analysis and that by Brune et al. 8

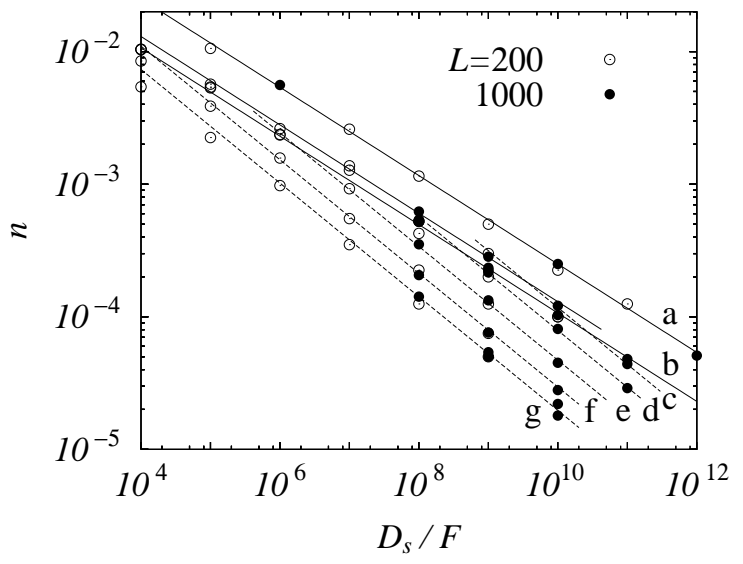

FIG. 3: Double logarithmic plot of the island density $n$ at the coverage $\theta=0.1 \mathrm{ML}$ versus the surface diffusion constant normalized by the deposition rate $D_{s} / F$. The values of edge and corner diffusion constants, $D_{e}$ and $D_{c}$, are (a) $D_{e}=D_{s}$ and $D_{c}=0$ (no corner diffusion), (b) $D_{e}=D_{c}=0$ (without edge and corner diffusion), (c) $D_{e}=D_{c}=10^{-4} D_{s}$, (d) $D_{e}=D_{c}=10^{-3} D_{s}$, (e) $D_{e}=D_{c}=10^{-2} D_{s}$, (f) $D_{e}=D_{c}=10^{-1} D_{s}$, and (g) $D_{e}=D_{c}=D_{s}$. In (a) the horizontal axis is displaced to the right by a factor 10 , for a visual purpose. Lines represent scaling behaviors as $n \propto\left(D_{s} / F\right)^{-\chi}$. An exponent $\chi$ for straight lines is $\chi=1 / 3$, and for dashed lines $\chi=3 / 7$.

$n=0.27\left(D_{s} / F\right)^{-1.027 / 3}$ for the DLA cluster in kMC simulation at the coverage $0.12 \mathrm{ML}$. For the DLA, in fact, another scaling law $n \propto\left(D_{s} / F\right)^{-2 /\left(4+D_{f}\right)}$ is proposed, where $D_{f}=1.71$ is the fractal dimension $24,25.26$ From the present simulations of sizes up to $1000^{2}$, we cannot descriminate which of the two scaling laws is superior.

For the case with a large edge diffusion $D_{e}=D_{s}$ but without corner diffusion $D_{c}=0$, islands have cross shapes as in Fig 2(i). Even for this situation, the density $n$ is compatible with a scaling law $n=0.25\left(D_{s} / F\right)^{-1 / 3}$, as is shown in Fig 3 (a). The plot is shifted by a factor 10 to the right for the visual purpose. Otherwise, data overlaps almost completely with those for fractal islands, Fig[3(b).

With or without edge diffusion, the island density is found to follow the same scaling relation with the surface diffusion constant $D_{s} / F$, obtained by a simple rate equation theory ${ }^{20.21}$ Inclusion of the corner diffusion, however, drastically changes the situation. By keeping $D_{e}=D_{c}$ but at different ratio to the surface diffusion as (c) $D_{e} / D_{s}=D_{c} / D_{s}=10^{-4}$, (d) $10^{-3}$, (e) $10^{-2}$, (f) $10^{-1}$, and $(\mathrm{g}) 1$, the relation between the island density $n$ and the surface diffusion constant $D_{s} / F$ varies as shown in Fig 3(c)-(g). For (c) the island density at low $D_{s} / F \leq 10^{10}$ is higher than that for (b) with fractal islands by about 20 percent, $n=0.28\left(D_{s} / F\right)^{-1 / 3}$. The density increase is due to the compactness of the islands, as shown in the surface configuration in Fig 4 (b) for $D_{e} / D_{s}=10^{-4}$, compared to the fractal islands in 


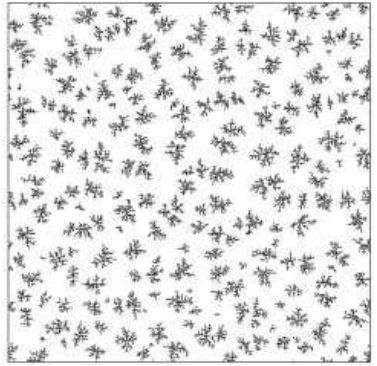

(a)

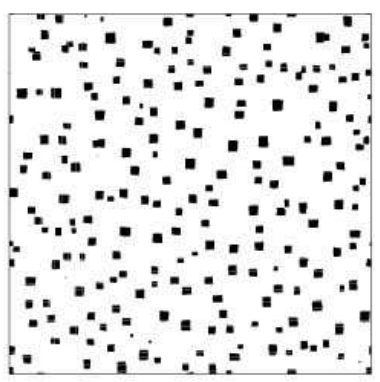

(c)

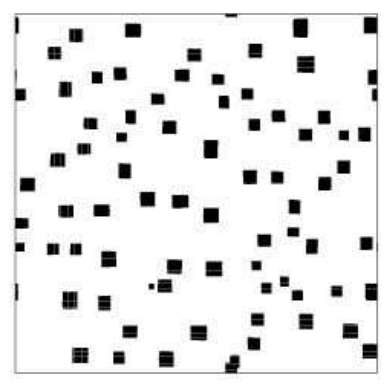

(e)

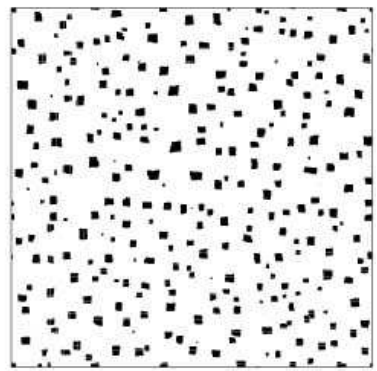

(b)

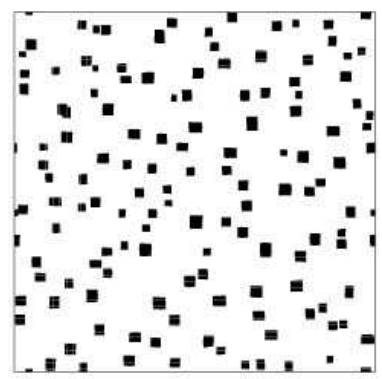

(c)

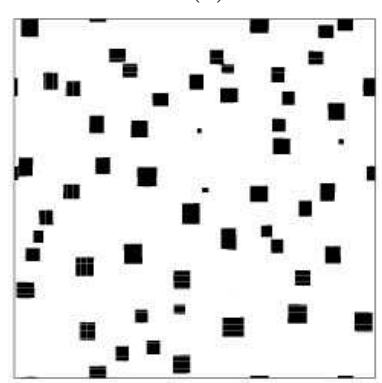

(f)
FIG. 4: Islands on a substrate of a size $1000^{2}$ at a coverage $\theta=0.1 \mathrm{ML} . D_{s} / F=10^{9}$ and $D_{e}=D_{c}$. The ratio of the corner to surface diffusion constant $D_{c} / D_{s}$ is (a) 0 , (b) $10^{-4}$, (c) $10^{-3}$, (d) $10^{-2}$, (e) $10^{-1}$, and (f) 1 .

Fig目 (a) for $D_{c}=0$. There are more space for adatoms to nucleate new compact islands. As for the $D_{s}$ dependence, the island density follows the same scaling law with an exponent $1 / 3$, unless $D_{s}$ takes the largest value $D_{s} / F=10^{11}$. There, the density becomes less than that of fractal islands, Fig 3 (b).

As $D_{e}$ and $D_{c}$ increase further from Fig [3(d) to (g), the island density starts to deviate from the curve Fig (3) c) at smaller $D_{s} / F$. For the case Fig B(d) with $D_{e} / D_{s}=D_{c} / D_{s}=10^{-3}$, deviation takes place at about $D_{s} / F=10^{9}$, for (e) at about $D_{s} / F=10^{7}$. For (f) and (g) with larger $D_{e}=D_{c}$, the island density remains always less than that of fractal islands with the same $D_{s}$. This tendency of density decrease is obvious by plotting the surface configurations in Fig 4 There, the coverage $\theta=0.1 \mathrm{ML}$ and the surface diffusion constant $D_{s} / F=10^{9}$ are the same, but the edge and corner diffusion constants, $D_{e} / D_{s}=D_{c} / D_{s}$, are varied. On in- creasing $D_{c}$ from Fig 4 (b) to (f), the number of islands decreases and the island size increases.

From Fig B(c) to (g), the island density $n$ seems to satisfy a new scaling relation to the surface diffusion constant $D_{s} / F$ as $n \propto\left(D_{s} / F\right)^{-\chi}$ with an exponent $\chi \approx 0.43$, when the density $n$ is lower than that of fractal islands. In Fig 3 we depict fitting lines with an exponent $\chi=3 / 7$, which is expected from the critical mobile island size $i^{*}=3$ and from eq.(2). By fitting the island density in the scaling form with an exponent $3 / 7$ as

$$
n=A\left(\frac{D_{s}}{F}\right)^{-3 / 7}
$$

the coefficient $A$ depends on the peripheral diffusion constant $D_{c}=D_{e}$. By plotting $A$ versus $D_{c} / D_{s}$ in double logarithmic way as in Fig 5 the coefficient $A$ is found to be proportional to $\left(D_{c} / D_{s}\right)^{-0.195}$.

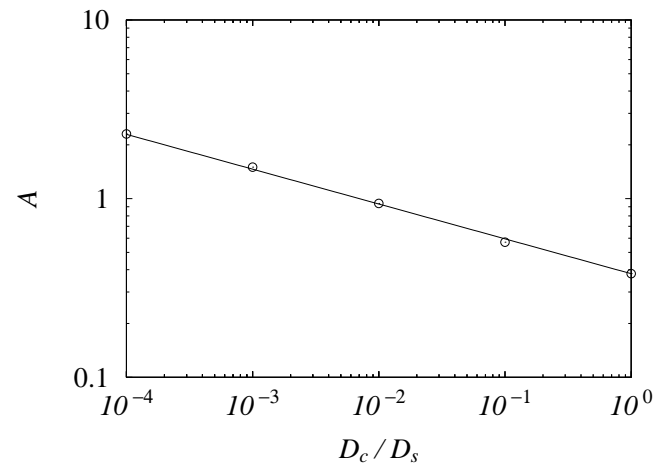

FIG. 5: The proportionality coefficient $A$ as a function of the corner diffusion constant $D_{c}$, which is equal to the edge diffusion constant $D_{e}$. The line represents the curve $A=$ $0.38\left(D_{c} / D_{s}\right)^{-0.195}$.

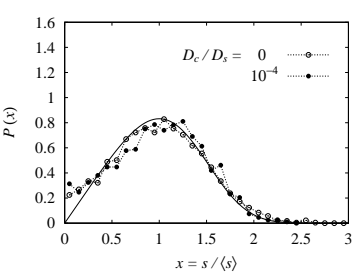

(a)

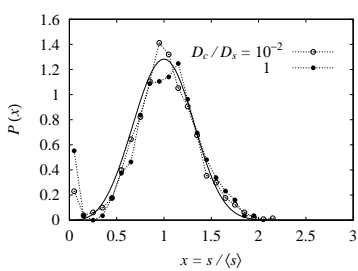

(b)
FIG. 6: Island size distribution with (a) low and (b) high corner diffusion constant, $D_{c}$.

From Fig 4 , one further notices that as the corner diffusion increases, not only the total number of islands but also the island size distribution changes $\stackrel{31.32}{=}$ This tendency is apparent by plotting island size distribution in Fig[6] Among the total $N_{i}=n L^{2}$ islands, there are $N(s)$ islands of a size $s$. Since the average island size $\langle s\rangle$ changes as $D_{c}$ varies, the distribution of islands with a normalized size $x=s /\langle s\rangle$ has the relevant concern. We 
define the probability $P(x) \Delta x$ as the number of islands with sizes between $x$ and $x+\Delta x$ devided by the total number of islands $N_{i}$ with $\Delta x=0.1$. For each $D_{c}$, size distribution of 10 samples are averaged. It is normalized as $\int_{0}^{\infty} d x P(x)=\int_{0}^{\infty} d x x P(x)=1$, and corresponds to the scaled number density as $N(s)=\left(\theta /\langle s\rangle^{2}\right) P(s /\langle s\rangle)$ at a coverage $\theta \frac{31.32}{1}$ At low $D_{c}$ 's as $D_{c}=0$ and $10^{-4} D_{s}$ in Fig [ (a), island sizes are widely spread with a broad peak such that there are very large islands with sizes about 2.5 times the average size. This is the typical size distribution for $i^{*}=1{ }^{31.32}$ On the contrary, at high $D_{c}$ 's the island size distribution has a narrow peak around the average size, and extends less than twice the average size in the region of large sizes. This is the typical size distribution for $i^{*}>1,27.31 .32$ When the clusters smaller then $i^{*}$ are dissociable, the island size distribution is proposed ${ }^{32}$ to have the form

$$
P_{i^{*}}(x)=C_{i^{*}} x^{i^{*}} \exp \left(-i^{*} a_{i^{*}} x^{1 / a_{i^{*}}}\right)
$$

with

$\frac{\Gamma\left[\left(i^{*}+2\right) a_{i^{*}}\right]}{\Gamma\left[\left(i^{*}+1\right) a_{i^{*}}\right]}=\left(i^{*} a_{i^{*}}\right)^{a_{i^{*}}}, \quad$ and $\quad C_{i^{*}}=\frac{\left(i^{*} a_{i^{*}}\right)^{\left(i^{*}+1\right) a_{i^{*}}}}{a_{i^{*}} \Gamma\left[\left(i^{*}+1\right) a_{i^{*}}\right.}$,

where $\Gamma$ means the Gamma function. The probabilities corresponding for $i^{*}=1$ and $i^{*}=3$ are drawn in Fig 6 (a) and (b) by continuous curves, respectively, and fit quite well with the simulation data, even though the dissociation is not allowed there. In fact, the similar size distributions with mobile clusters are obtained in the previous Monte Carlo simulation ${ }^{27}$ There, however, no peripheral diffusion is granted, and the grown islands had fractal structures, in constrast to our square shaped clusters.

\section{DISCUSSIONS AND CONCLUSIONS}

We have seen that a large corner diffusion influences the island number density and the size distribution drastically. Without or weak corner diffusion, the island density varies in proportion to $\left(D_{s} / F\right)^{-\chi}$ with an exponent $\chi=1 / 3$, irrespective of the island morphology. This means that the nuclei are formed at the initial stage of deposition, as soon as the two adatoms collide with each other. They are immobile and act as a center of nucleation. Therefore, the randomness of the two-adatom encounter is frozen in. After sufficient density of nuclei is formed, further deposited adatoms are incorporated into the preexisting clusters. They develope into various shapes, depending on the edge and corner diffusion constants. Since the nucleation takes place at random, the island sizes are distributed rather broad, reflecting the randomness in the island separation. In any case, this senario permits the island density to be independent of the morphology: The island density is determined long before the island shape appears.

With a large corner diffusion, on the other hand, inspections of the time evolution reveal that clusters of small sizes $i \leq 3=i^{*}$ become mobile. Since atoms in a cluster smaller than the critical size $i^{*}=3$ can move around each other via the corner diffusion, small clusters migrate around randomly on the substrate surface. During this cluster diffusion, they coagulate with isolated atoms and as a result the island density decreases. Of course, islands larger than the size $i^{*}=3$ can migrate in some configurations, but when compact islands with doubly or more bonded atoms are formed, they cease migration on the substrate surface, and start to act as a nucleation center. During the further stage of cluster growth, the adsorbed atoms form small mobile clusters and are incorporated into islands in group.

This situation is treated by Villain et al ${ }^{24}$ in terms of the rate equation, and the island density $n$ is expected to follow the scaling relation eq.(2). Since the trimer can migrate on the surface, $i^{*}=3, n \propto F^{3 / 7}$ is expected. This is the reason why we fitted the density with a scaling exponent $\chi=3 / 7$ in Fig 3 This explains the $F$ dependence of $n$, but as for the dependence on diffusion constants, we have to know $D_{2}$ and $D_{3}$ in terms of the present edge and corner diffusion constants, $D_{e}$ and $D_{c}$.

There is an exact calculation by Sanchez et al ${ }^{35}$ of diffusion constants of small clusters mediated by a peripheral diffusion. The result is simple for a dimer and a trimer on a square lattice as

$$
D_{2}=\frac{D_{c}}{2}, \quad D_{3}=\frac{D_{e} D_{c}}{3\left(D_{e}+D_{c}\right)} .
$$

For a dimer to diffuse, one of the adatoms which compose dimer has to cross round the corner. For a trimer, for example, in an extended form to move an edge atom has to cross the corner and to slide on the edge to another corner. Without edge diffusion, the center of mass of a trimer cannot move. In the case shown in Fig $3 D_{e}=D_{c}$ and thus $D_{3}=D_{c} / 6$. Inserting these exact results into the expression (21) obtained by rate equation, the island number density is expected to follow the relation

$$
n \propto\left(\frac{F^{3}}{D_{s} D_{c}^{2}}\right)^{1 / 7}
$$

If this scaling holds, the coefficient $A$ in eq. (33) should be proportional to $A \propto\left(D_{c} / D_{s}\right)^{-2 / 7}=\left(D_{c} / D_{s}\right)^{-0.286}$, instead the exponent -0.195 found in Fig 5 Therefore, the dependence of $n$ on cluster diffusion constant does not seem to be properly given by the rate equation.

To check the scaling behavior of the island density with the crossover from the monomer- to the trimerlimited form, let us study the scaled island density $\tilde{n}=$ $n\left(D_{s} / F\right)^{1 / 3}$. It should be constant around 0.23 for small edge and corner diffusion constnat $D_{e}=D_{c}$. For large $D_{e}=D_{c}$, it should be proportional to $\left(D_{s}^{2} F / D_{c}^{3}\right)^{2 / 21}$, according to the result of the rate equation analysis (2). Therefore, we try the plot $\tilde{n}$ versus $\tilde{F}_{1}=D_{s}^{2} F / D_{c}^{3}$, but the data does not collapse on a single universal curve.

From our simulation result, the island density behaves as $n=A\left(D_{s} / F\right)^{-3 / 7}$ with an $D_{c^{-}}$-dependent coefficient 


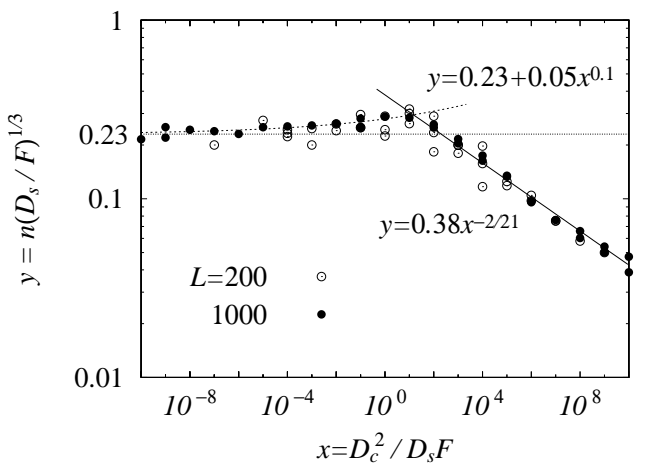

FIG. 7: The scaled island density $y=n\left(D_{s} / F\right)^{1 / 3}$ as a function of the scaled corner diffusion constant $x=D_{c}^{2} / D_{s} F$. The dashed curve represents small $x$ asymptotic, $y=0.23+$ $0.05 x^{0.1}$, and the continuous line the large $x$ asymptotic form $y=0.38 x^{-2 / 21}$.

$A=0.38\left(D_{c} / D_{s}\right)^{-0.195}$ for large $D_{c}$, as depicted in Fig 4 Since the exponent 0.195 is approximately equal to $4 / 21$, the density is expected to be scaled by the variable $x=D_{c}^{2} / F D_{s}$ at a large $D_{c}$ more properly. The scaling plot is shown in Fig 7 for a wide range of combinations of diffusion constants as $D_{s} / F=10^{5} \sim 10^{11}$ and $D_{e}=D_{c}=10^{-10} D_{s} \sim D_{s}$, and one observes a nice data collapse on a universal curve. It is fitted as

$$
n\left(\frac{D_{s}}{F}\right)^{1 / 3}= \begin{cases}0.23+0.05 x^{0.1} & \text { for } x \rightarrow 0 \\ 0.38 x^{-2 / 21} & \text { for } x \rightarrow \infty\end{cases}
$$

as shown in Fig 7

The effect of the edge and corner diffusion during the homoepitaxial nucleation growth on a singular surface is now summarized as follows. As for the island morphology, these peripheral diffusion makes the island regular and compact. With only the surface diffusion, the irreversibly nucleated islands have a fractal shape characteristic to DLA. With the edge diffusion, islands become thick and compact. The corner diffusion affects the shape anisotropy. One may typically say that without the corner diffusion dendritic needle pointing in the $\langle 10\rangle$ direction appear, whereas with it squares with $\{10\}$ faces appear.

The density of islands is mainly determined before the shape appears. Without or with a weak corner diffusion, the critical island size is $i^{*}=1$, and the density $n$ is related to the surface diffusion constant $D_{s}$ normalized by the deposition rate $F$ as $n \propto\left(D_{s} / F\right)^{-1 / 3}$, irrespective of island morphology. With a large corner diffusion constant, on the other hand, small clusters $i \leq 3$ can migrate randomly on the substrate surface, and another scaling relation $n \propto F^{3 / 7} / D_{s}^{5 / 21} D_{c}^{4 / 21}=\left(F / D_{s}\right)^{3 / 7}\left(D_{s} / D_{c}\right)^{4 / 21}$ is found appropriate. The flux $F$-dependence agrees with the rate equation theory with $i^{*}=3$, but the dependence on diffusion constants $D_{c}$ contradicts the theory. The reason of this discrepancy is not clear. But, since the rate equation is a mean-field type approximation which neglects spatial correlation, it may be fortuitous that $F$ dependence agrees in the simulation and the rate equation theory.

Recently, the mobility of large clusters is proposed to be very effective in selecting the island size and in arranging islands in order in heteroepitaxial growth ${ }^{36}$ Our model allows the motion of only small clusters less than the size $i^{*}=3$ by the corner diffusion, and some size selection is observed. Due to the absence of any direct interaction between islands, however, the ordering in island positions cannot be achieved. Our model neither contains the size-limiting effect, and the coarsening takes place as the corner diffusion increases.

\section{Acknowledgments}

This work is supported by Grant-in-Aid for Scientific Research from Japan Society of the Promotion of Science. The author is benefited from the inter-university cooperative research program of the Institute for Materials Research, Tohoku University.
* Electronic address: yukio@rk.phys.keio.ac.jp

1 R.Q. Hwang, J. Schroder, C. Gunther, and R.J. Behm, Phys. Rev. Lett. 67 (1991) 3279.

2 T. Michely, M. Hohage, M. Bott, and G. Comsa, Phys. Rev. Lett. 70 (1993) 3943.

3 J.A. Stroscio, D.T. Pierce, and R.A. Dragoset, Phys. Rev. Lett. 70 (1993) 3615.

${ }^{4}$ H. Brune, C. Romainszyk, H. Röder, and K. Kern, Nature (London) 369 (1994) 469.

5 H. Brune, H. Röder, C. Romainszyk, C. Boragno, and K. Kern, Appl. Phys. A 60 (1995) 167.

${ }^{6}$ M. Hohage, M. Bott, M. Morgenstern, Z. Zhang, T. Michely, and G. Comsa, Phys. Rev. Lett. 76 (1996) 2366.

7 Y. Li, M.C. Bartelt, J.W. Evans, N. Waelchli, E. Kamp- shoff, and K. Kern, Phys. Rev. B 56 (1997) 12539.

8 H. Brune, G.S. Bales, J. Jacobsen, C. Boragno, and K. Kern, Phys. Rev. B 60 (1999) 5991.

9 T.A. Witten and L.M. Sander, Phys. Rev. Lett. 47 (1981) 1400.

10 T. Irisawa, M. Uwaha and Y. Saito; Europhys. Lett. 30 (1995) 139.

11 G.S. Bales and D.C. Chrzan, Phys. Rev. B9 (1994) 6057.

12 Z. Zhang, X. Chen and M.G. Lagally, Phys. Rev. Lett. 73 (1994) 1829.

13 G.S. Bales and D.C. Chrzan, Phys. Rev. Lett. 74 (1995) 4879 .

14 S. Ovesson, A. Bogicevic, and B.I. Lundqvist, Phys. Rev. Lett. 83 (1999) 2608. 
15 J.G. Amar, Phys. Rev. B60 (1999) R11317.

16 J. Zhong, T. Zhang, Z. Zhang, and M. Lagally, Phys. Rev. B 63 (2001) 113403.

17 C.R. Stoldt, A.M. Cadilhe, C.J. Jenks, J.-M. Wen, J.W. Evans, and P.A. Thiel, Phys. Rev. Lett. bf 81 (1998) 2950.

18 O. Pierre-Louis, M.R. D'Porsogna, and T. Einstein, Phys. Rev. Lett. 82 (1999) 3661.

19 M.V. Ramana Murty and B.H. Cooper, Phys. Rev. Lett. 83 (1999) 352.

20 J.A. Venables, Phil. Mag. 27 (1973) 697.

21 S. Stoyanov and D. Kaishiev, Curr. Top. Mater. Sci. 7 (1981) 69.

22 J.A. Venables, G.D.T. Spiller, and M. Hanbücken, Rep. Prog. Phys. 47 (1984) 399.

23 T. Irisawa, Y. Arima, and T. Kuroda; J. Cryst. Growth 99 (1990) 491.

24 J. Villain, A. Pimpinelli, and D. Wolf, Comments Cond. Mat. Phys. 16 (1992) 1.

25 J. Villain, A. Pimpinelli, L.-H. Tang, and D. Wolf, J. de Physique I 2 (1992) 2107.
26 L.-H. Tang, J. de Physique I 3 (1993) 935.

27 I. Furman and O. Biham, Phys. Rev. B 55 (1997) 7917.

28 M.C. Bartelt and J.W. Evans, Phys. Rev. B46 (1992) 12675.

29 S. Liu, L. Bönig, and H. Metiu, Phys. Rev. B 52 (1995) 2907.

30 P.A. Mulheran and D.A. Robbie, Phys. Rev. B 64 (2001) 115402.

31 M.C. Bartelt, S. Günther, E. Kopatzki, R. j. Behm, and J.W. Evans, Phys. Rev. B 53 (1996) 4099.

32 J.G. Amar and F. Family, Phys. Rev. Lett. 74 (1995) 2066.

33 W.F. Berg, Proc. Roy. Soc. A164 (1938) 79.

34 K.J. Caspersen, C.R. Stoldt, A.R. Layson, M.C. Bartelt, P.A. Thiel, and J.W. Evans, Phys. Rev. B 63 (2001) 085401.

35 J.R. Sanchez and J.W. Evans, Phys. Rev. B 59 (1999) 3224.

${ }^{36}$ F. Liu, A.H. Li, and M.G. Lagally, Phys. Rev. Lett. 87 (2001) 126103. 